\title{
Evaluation and Comparative Study of Structure Free Aggregation Techniques for Duty Cycled Wireless Sensor Networks
}

\author{
Sukhwinder Singh Sran \\ YCOE, Punjabi University \\ Guru Kashi Campus, Talwandi Sabo \\ Bathinda, Punjab, India-151302
}

\author{
Lakhwinder Kaur \\ Department of Computer Engineering \\ Punjabi University, Patiala \\ Punjab, India - 147002
}

\begin{abstract}
For delay sensitive event based applications, structure free data aggregation approach has been found to be more suitable because of the reduced structure maintenance overhead. However, in the literature, it has been observed that increasing the delay at intermediate sensor node increases aggregation and the event notification time. The objective is to investigate various structure free aggregation schemes in terms of aggregation gain, event notification time and the overall energy consumption. To achieve this objective, a comparative study of various structure free aggregation techniques is presented. First, we investigate the potential of aggregation in different structure free aggregation techniques for duty cycled sensor networks. Then the performance of the aggregation techniques is demonstrated with the help of simulations on Cooja network simulator in Contiki operating system. From the results, it has been observed that Tuned delay based aggregation scheme reduces energy consumption (upto 13\%) as compared to Collect protocol. Moreover, it reduces the worst event notification time (upto $20 \%$ ) in comparison to random delay based aggregation scheme.
\end{abstract}

\section{General Terms}

Wireless sensor networks, Data aggregation

\section{Keywords}

Structure free aggregation, Energy efficient routing, Event notification time, Duty cycling, Aggregation Delay

\section{INTRODUCTION}

Sensor nodes have limited energy, transmission capability, computing power and are often non-chargeable. These nodes are battery enabled and mostly deployed over isolated or hostile environments. Therefore, it is essential for the network to stay alive for prolonged duration to achieve good network lifetime. Sensor nodes gather data from their surroundings, process it locally and finally transmit the data packets to a designated node called as sink [1]. Most of the time, correlated data packets are generated by several sensor nodes from a sensing region. The correlated data is purely transmission overhead and increases the communication cost, which may deplete the batteries of sensor nodes rapidly [24]. To minimize this overhead, data aggregation or in-network processing is an elegant solution. Data aggregation focuses on reducing the number of transmissions required to report gathered data or events. By using basic aggregation functions such as Min, Max, Sum, Count, Avg etc., the network traffic can be minimized [6].

Many energy efficient routing protocols use aggregation structure such as clusters, trees (such as MST or SPT) or small chains to aggregate data in the network. These techniques are suitable for data gathering applications and use fixed structure to transmit entire data to the final destination. Whenever there is a topological change or failure in network, the structure needs maintenance or to be reconstructed again. The structured aggregation approach incurs high structure maintenance overhead in dynamic scenario. On the other hand, structure free data aggregation approach is specifically suitable for event based applications in dynamic networks (where event triggering time and location is dynamic). This approach uses anycasting to transmit data to sink and use early aggregation to reduce the volume of data [9] In addition, structure free data aggregation is observed as an efficient approach for mobile WSN (where sensor nodes or the target region is not stationary).

Sensor nodes primarily consume energy for data communication (i.e. number of transmissions or receptions) and an equal amount of energy is consumed in idle listening state. In idle listening, sensor node does not perform any useful work and depletes the node's energy. To minimizes this consumption, low duty cycling mechanism is widely accepted solution. The low duty cycling technique can reduce energy consumption tremendously, by minimizing the idle listening. The sensor nodes do not perform any useful work during idle listening and wastes almost one third of the residual energy of sensor node. In low duty cycling, sensor nodes remain in sleep state for most of the time and turn on to active state periodically for few milliseconds to listen for the events. The basic idea is to save energy by managing the active time of nodes properly and to maximize their sleep time while achieving application goals such as low latency or high throughput. Moreover, this scheme allows the sensor nodes to optimize and balance their energy consumption [14, 21].

This paper contributes by providing a comparative analysis of structure free aggregation strategies for duty cycled WSN. The basic mechanism is to exploit the sophisticated delay at each aggregation point for aggregating data in the techniques.

The contents of the paper are organized in the following man- 
ner. Section-2 discusses various existing structure free data aggregation and dynamic aggregation aware routing techniques. The MAC layer protocol (ContikiMAC) and routing layer protocol (Collect) used in Contiki operating system are presented in section3 Section-4introduces the network and energy model used for the aggregation aware techniques. The discussion of the various structure free aggregation schemes is provided in section-5 The next section-6 demonstrates the comparative study of the simulation results for the Normalized, Tuned and Randomized aggregation techniques and Collect protocol. The conclusion and future scope of the work are provided in section-7

\section{RELATED WORK}

Several existing data aggregation protocols use fixed aggregation structure such as clustering, aggregation trees and multiple chains for aggregating data. These techniques use static route to forward or aggregate data without considering the location, residual energy or other routing metrics of the deployed nodes for making decision to construct a routing path dynamically. Some traditional data aggregation protocols that use such routing or aggregation mechanism are LEACH [13], PEGASIS [18], improved PEGASIS [19], PEDAP [29], COSEN [28], BERP [15], TL-LEACH [20], TBLEACH [12], CHIRON [4], CREEC [25], EECF [17], EA-COSEN [26] etc. These protocols are suitable for data gathering applications and performs well in static wireless sensor networks.

For event based applications, structured data aggregation techniques suffer from topology maintenance overhead specially in dynamic network situations. In unstructured networks, dynamic routing is used to forward data for achieving efficient data aggregation without experiencing maintenance overhead of the structure. Various protocols use dynamic routing to forward and aggregate data to the final destination that are explained as under:

The information fusion techniques are generally used to fuse the data gathered from the surrounding. In these techniques, the role of one node is fixed in advance (proactive) to fuse sensory information even if there is no event in the network. Hence, assigning role in advance may deplete the energy of designated nodes quickly. To elevate this problem, Information Fusion based Role Assignment technique was developed by Nakamura et al. [23]. Information Fusion based Role Assignment technique (InFRA) assigns the role to the designated node only when an event is detected in the network (On-demand). The results confirms that InFRA reduces energy consumption and increases aggregation in the network.

Dynamic Data Aggregation Aware Routing Protocol (DDAARP) builds the routing tree dynamically to collect the data of the events (having short duration). For such events, construction of the static structure for aggregation is costly and requires large number of messages, to set up the routing tree. To enhance aggregation, DDAARP constructs the routes having higher data aggregation rate, maximizing the overlapping routes and minimizing overhead to build routing tree. The results provided in paper indicates that DDAARP performs better than InFRA and DAARP protocol in terms of scalability, communication cost, data delivery and aggregation rate [31].

Data Aware Anycasting and Randomized Waiting- DAA+RW protocol routes the data packets dynamically by using anycasting. Fan et al. was the first to use anycasting in sensor networks for developing Data Aware Anycasting and Randomized Waiting (DAA and $\mathrm{RW})$ protocol [9]. DAA + RW was the first structure free data aggregation protocol suitable for those applications, where it is difficult to locate the center of event. DAA + RW protocol achieves good performance in terms of aggregation gain without using any fixed structure. But this protocol is not scalable i.e. it does not guarantee good aggregation gain as network grows and incur long delay in large networks.

An another fuzzy based structure free aggregation technique was proposed by Dietzel et al. [5] for Vehicle Ad hoc Networks. This approach is suitable for those applications, where periodic data dissemination takes place around a large field like traffic information system. To integrate highly correlated data, data aggregation takes place based on fuzzy reasoning. This scheme performs better as compared to flooding, in terms of bandwidth and congestion [5]. Dynamic and Scalable Tree- DST: Dynamic and Scalable Tree protocol forwards data along the routing paths which have higher chances of data aggregation. To balance the energy consumption within network, this protocol uses dynamic aggregation structure for relaying data towards sink. Hence, the routing tree is independent of the order of events and changes each time on the occurrence of an event. To establish the routing path, DST uses minimum number of control packets to reduce energy consumption. DST outperforms in terms of scalability, communication cost and the routing structure cost in contrast to InFRA and DAARP [32].

Data aggregation supported by dynamic routing- DASDR: To perform energy efficient routing and aggregation in dynamic network situations, DASDR scheme uses dynamic aggregation structure in contrast to static routing techniques. To ensure the delivery of every data packet at sink, concept of depth potential field is implemented that forcefully forwards each data packet from source node to one of their neighbors and finally, data packet is delivered to the destination. In addition, each neighbor maintains queue to buffer data packets and may try to spatially converge more data as compared to existing techniques. The comparative results presented in paper shows that DASDR is more energy efficient, having higher aggregation ratio and more scalable than CT and SPT protocol [34].

Real Time Data Aware Anycasting and Judiciously Waiting: Yousefi et al. [33] found that in dense networks, data aggregation reduces energy consumption by eliminating default repetitiveness in sensed data. Yousefi et al. proposed structure free real time data gathering techniques, using spatial and temporal convergence of data, called as Real time Data aware Anycasting and Judiciously Waiting. These techniques are evaluated using extensive simulations in ns2, based on delay, power dissipation, reductions in communication traffic and discarded packets during transmission to sink. The performance of Real time Data aware aycasting and Judiciously waiting protocol is much better than conventional protocols like SPEED, DASDR and DAA+RW [33].

dYnamic scalablE tree Aware of Spatial correlaTion- YEAST: To get accurate data, sensor nodes are densely deployed in several accuracy sensitive applications. In these networks, neighboring nodes are likely to collect correlated and redundant data which may enhance energy consumption in sensor network. In YEAST, sensing field is divided into several correlated regions and one of the node called as representative node is elected to aggregate and forward the data to the next hop. Nodes detecting the same event are grouped in one correlated region and each correlated region have only one representative node whose role is rotated after every round to balance the energy consumption among the nodes. YEAST is evaluated in terms of accuracy of event detection which is $95 \%$ and can save $75 \%$ residual energy as compared to traditional data gathering techniques [30].

Structure Free and Energy Balanced-SFEB: Chao et al.[3] proposed a novel approach called as structure free and energy balanced data aggregation for sensor networks. This scheme overcomes the drawbacks of DAA + RW protocols and is designed for spatial and temporal convergence of the data. Moreover, the aggregator is 
elected dynamically using light weight aggregator election mechanism. For early aggregation, data aggregator are selected as soon as possible in setup phase. To balance energy consumption, this technique implements scheduled data aggregation among the neighbors of the aggregator. Simulated results confirm that this technique improves aggregation gain and consume less energy [3].

Aggregation Aware Early Event Notification Technique-AAEENT: To improve the event notification delay and aggregation gain, AAEENT [27] scheme is proposed. This technique uses aggregation aware reliable routing policy to relay the data in the network. The simulated results confirm the superiority of the technique in terms of aggregation, event notification delay and power consumption in comparison to existing aggregation schemes.

\section{PRELIMINARIES}

An idle listening is useless radio state in which sensor nodes consumes almost one third of the total energy of a sensor node as discussed in section-1. To minimize this energy wastage, low duty cycling is one of simple and efficient approach for WSNs. The low duty cycled sensor networks are categorized as synchronous and asynchronous low duty cycled sensor network. In synchronous low duty cycled WSN, source and destination nodes have synchronized transmit and receive schedules. Therefore, sender transmits data only when the receiver is checking channel to receive data packet. In asynchronous low duty cycled WSN, nodes remain in sleep state to save the power. The energy consumption is reduced tremendously in this dormant phase. To detect an activity in sensor network, nodes periodically wakeup to sample the wireless channel. Whenever any node detects radio signal in the network, it turns its radio on and starts receiving the data packet. The performance (in terms of end to end delay and aggregation gain) of the synchronous low duty cycled WSN is significantly higher than that of asynchronous schemes. But synchronous schemes are not suited well for dynamic sensor networks where it is difficult to maintain synchronized schedules for source and destination. The aggregation strategies are designed to work above the ContikiMAC protocol [7] and are implemented as a part of network layer. To understand the basic functionality of ContikiMAC- duty cycled MAC protocol and Collect routing protocol, an introductory information is provided in the following sub-sections.

\subsection{Low Duty Cycled WSN- ContikiMAC}

The ContikiMAC protocol is designed as simple and efficient protocol for low power duty cycled WSNs. It is more power efficient than its ancestor protocol: X-MAC [2]. It uses asynchronous duty cycled schedules for communicating with neighbors and other nodes. The strict timing constraints and fast sleep mechanism in contikiMAC ensures that it is power efficient protocol. By learning the wake-up schedule of the receiver, it optimizes the transmissions.

\subsection{Contiki- Routing protocol (Collect)}

Many wireless sensor network applications use collection tree protocol (CTP) for reliable data delivery. The collection tree routing protocol is used to find an optimal path between a pair of wireless sensor nodes in highly dynamic environments. The various functions of CTP include: creation of routing tree, neighbor discovery, maintenance of neighbor tables, link estimation (using expected number to transmissions- ETX), adaptive beaconing (using few beacons to control traffic) and data path validation (by detection and recovery from path failures). Due to these features, this protocol is robust to failures and topological changes. The data

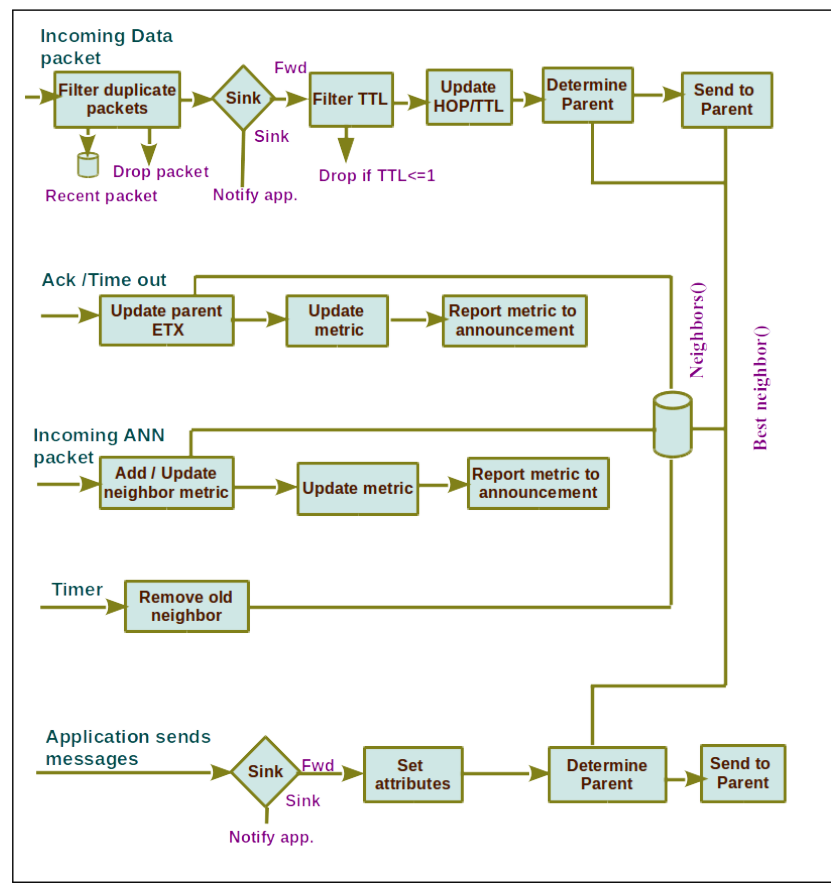

Fig. 1: Event Processing in Contiki- Collect protocol

path validation is one of the important module of Collect protocol [10, 11]. Firstly, the nodes construct virtual tree by organizing themselves for data transmission from source to sink. The rtmetric of the each node is updated based on the rtmetric of every new discovered node. The neighbor tables receive their entries by sending and receiving the announcement packets through the rime announcement primitive. For delivering data, Collect discovers reliable route by using expected transmission count in link estimation phase. The expected transmission count represents the number of transmissions required for successful delivery of data at the final destination. The value of the rtmetric assigned to the nodes decreases towards the sink and is minimum (zero) for the sink. Based on the ETX values, each node selects the best parent (having lesser ETX). Moreover, some control packets are used by the nodes to update rtmetric values to deal with topological change by using Trickle algorithm [16]. Figure 1 demonstrates the processing of data for various modules at different nodes in Collect protocol. The Trickle algorithm sends only the core updates to dynamically selected and advised paths in the network. Moreover, Collect discovers the routing loop and route inconsistencies, whenever there is a packet to transmit. These loops and route failures are repaired by route recovery module. So nodes will wait and transmit data over an updated and consistent path instead of dropping packet.

To transmit or receive data from the parent node, each node maintains a send queue to store recently forwarded or received packets. It is also used to eliminate the duplicate data packets received at a particular node. The receiver sends a positive acknowledgement for a successful data delivery. In case of failure, the data is retransmitted again. There is limit on the number of retransmissions at each node and after exceeding that limit, node drops the data packet. Several primitives used by CTP are demonstrated by figure 2 and are explained as under:

All the functions except unicast_send primitive are part of the Collect routing protocol. The unicast_send primitive is an inter- 


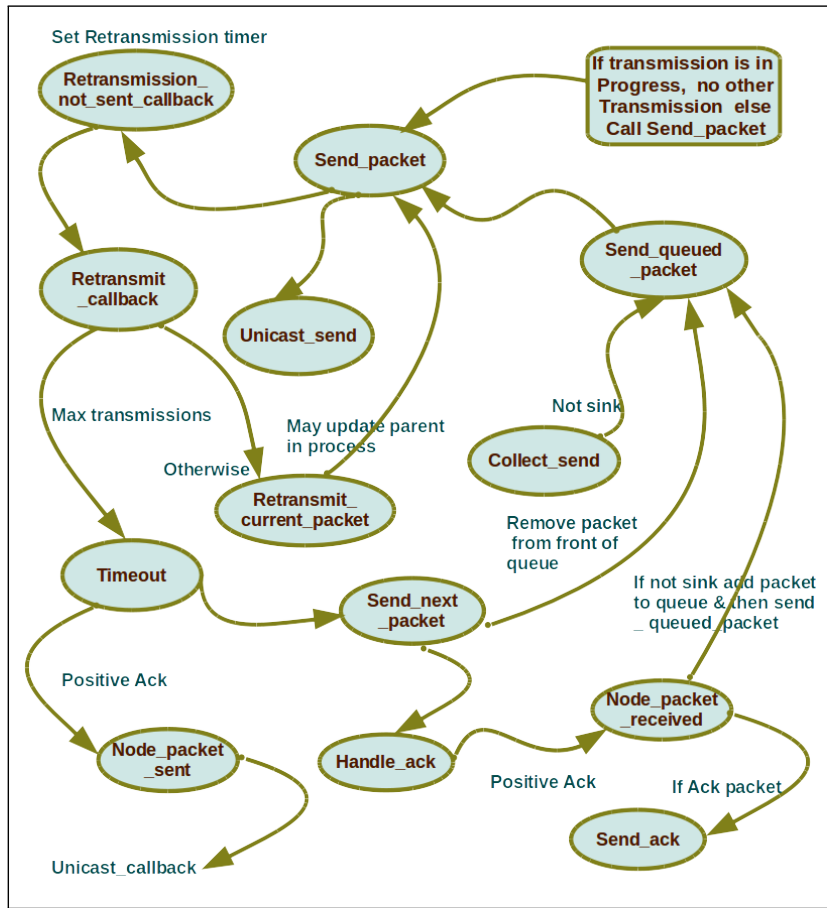

Fig. 2: Various activities and flow of data among them at a node in Collect

face between Contiki- Collect and ContikiMAC protocol. The collect_send is used as an interface for the application layer of the sensor node. The packet from the unicast layer is received at the node_packet_received function. Both functions collect_send and node_packet_received add packets to the packet queue and then call the function send_queued_packet to try forwarding the packet to the next parent if the current node is not the sink. The flow of data among various primitives in Contiki- Collect implementation is shown in figure 2

\section{SIMULATION SCENARIO}

The first sub-section presents the network scenario used for the simulation of the structure free data aggregation techniques. The energy model used to compute the energy consumed in different states is explained in the next sub-section.

\subsection{Network Model}

To test the performance of the aggregation schemes, 25 nodes are deployed over a grid of $100 \mathrm{~m}$ x $100 \mathrm{~m}$ by using Cooja network simulator. The nodes are low power wireless sensors devices (Tmote sky) and are homogeneous i.e. every node is equipped with similar resources. The transmission range allows each node to communicate with other nodes that are only one hop away. Each event has its own predefined radius and is spatially distributed. The internode separation, transmission and interference range is fixed for each node in advance. Each sensor node is using Contiki operating system to generate, process and to communicate data over the network. The network can detect, process and transmit different types of arbitrary events within the network. In this network scenario, 25 nodes are deployed to sense and track events, out of which 22 nodes are triggered by 6 different types of events. Each intermediate node holds the transmissions for short duration and tries to aggregate the data received from their immediate neighbors. The aggregated data is transmitted to next hop and the same procedure is repeated for the next hop until data is delivered to the final destination.

\subsection{Energy Model}

Sensor nodes are power deficient, therefore an efficient use of energy remains the key challenge in sensor networks. In this work, sensor nodes (Tmote sky) which are powered by two AA batteries are used to test the performance of the aggregation schemes. The power consumed by Tmote sky to process data by MPU (active mode and low power mode) is $5.4 \mathrm{~mW}$ and $0.163 \mathrm{~mW}$ respectively [22]. The radio contributes for maximum energy consumption to handle communication among the nodes in the network. Therefore, to minimize energy consumption, nodes use low duty cycled approach. A linear model is used by energy estimation module to compute energy consumed by sensor nodes [8]. The energy consumed by sensor nodes in different states is given below:

$E_{c p u}$ - Energy Consumed by MPU in Active Mode

$E_{l p m}$ - Energy Consumed MPU in Low Power Mode

$E_{t x}$ - Energy Consumed by radio in Transmit Mode

$E_{r x}$ - Energy Consumed by radio in Receive Mode

$E_{s_{i} l_{j}}$ - Sum of power consumed by all other components such as sensors and LEDs. where

$$
E_{s_{i} l_{j}}=\sum_{i=1, j=1}^{n} s_{i} l_{j}
$$

The value of $E_{s_{i} l_{j}}$ is negligible as compared to energy consumed by MPU and radio. The total energy consumption is denoted by $E_{t}$ is as under:

$$
E_{t}=E_{c p u}+E_{l p m}+E_{t x}+E_{r x}
$$

$C_{t}$ and $L_{t}$ - Amount of time that MPU is spent in active mode and low power mode,

$T_{t}$ and $R_{t}$ - Data transmission and reception time. Total MPU time (active + low power mode) $T_{m}=C_{t}+L_{t}$

$$
\begin{array}{r}
E_{c p u}=\frac{1.8 \times C_{t}}{T_{m}} \times V \\
E_{l p m}=\frac{0.0545 \times L_{t}}{T_{m}} \times V \\
E_{t x}=\frac{17.7 \times T_{t}}{T_{m}} \times V \\
E_{r x}=\frac{20 \times T_{t}}{T_{m}} \times V
\end{array}
$$

Residual Energy of a node can be computed as:

$$
E_{\text {res }}=E_{t}+E_{\text {consumed }}
$$

\section{AGGREGATION STRATEGIES- NDDA, RDA AND TDA}

In this paper, Normal distribution delay (NDDA), Randomized delay (RDA) and Tuned delay (TDA) based structure free aggregation schemes are investigated for duty cycled WSN. The summarized information about these schemes is given in the following paragraphs. 


\subsection{Normal distribution delay based aggregation- NDDA}

The normalized delay at every aggregator is essential to reduce the end-to-end delay and energy consumption. In normal distribution delay based aggregation scheme, the aggregation delay is normalized among the sensor nodes. In this scheme, standard normal distribution is used to generate Gaussian random number for computing the aggregation delay. The procedure to generate Gaussian random number by using Box-Muller algorithm (with mean ' 0 ' and standard deviation ' 1 ') is given below:

Let $U_{1}$ and $U_{2}$ be two random number distributed uniformly among the interval [0,RAND_MAX].

$$
\begin{gathered}
U_{1}=\operatorname{rand}() *\left(1.0 / R A N D \_M A X\right) ; \\
U_{2}=\operatorname{rand}() *\left(1.0 / R A N D_{-} M A X\right) ; \\
Z_{1}=\sqrt{-2 \times \log U_{1}} \times \cos \left(2 \pi U_{2}\right) \\
Z_{2}=\sqrt{-2 \times \log U_{1}} \times \sin \left(2 \pi U_{2}\right)
\end{gathered}
$$

$Z_{1}, Z_{2}$ are normalized variable obtained by using standard normal distribution. This normalized form of delay helps in reducing the connectivity holes in the sensor field, specially near the sink. This uniform distribution of the aggregation delay helps in reducing the event notification time as well as energy consumption.

\subsection{Randomized delay based aggregation- RDA}

The amount of aggregation in this randomized delay based aggregation scheme depends on the aggregation delay assigned to an aggregator. In this scheme, pseudo random number generator is used to generate the random values that can be assigned to each aggregating node. The pseudo random integral number generator function $\operatorname{rand}()$ generates the numbers between a specified range ( 0 to RAND_MAX).

$$
a g g_{\text {delay }}=\operatorname{abs}(\operatorname{rand}() \% n)
$$

where $n \in\left[0, R A N D \_M A X\right]$. To improve aggregation gain, each aggregator must delays the transmissions to gather and aggregate more data from the descendant or neighboring nodes. To avoid the adverse effect on end to end to delay, this function is used to reinforce set of smaller values for every aggregator. Finally, the converged data is delivered to the sink through various aggregators. Due to randomness, this method does not distribute the values (aggregation delay) uniformly among the nodes in the networks.

\subsection{Tuned delay based aggregation- TDA}

In tuned delay based aggregation scheme, the objective is distribute the delay uniformly so that end to end delay can be minimized. In this technique, pre-determined aggregation delay introduced at each intermediate node for the aggregation. By tuning the pre-defined delay among the aggregators, the performance of the scheme is improved. Although it requires extensive simulations, but the significant performance can be achieved over the existing randomized delay based aggregation scheme.

$$
a g g_{\text {delay }}=\frac{\delta}{\rho}
$$

where $\rho$ is pre-defined delay for each intermediate node and $\delta$ is tunable parameter for this scheme.

\section{DISCUSSION OF RESULTS}

The performance of the Normalized, Randomized and Tuned delay based aggregation schemes is measured by using three parameters that are explained as under:

Aggregation Gain: It is the number of data packets aggregated by the intermediate nodes (aggregators) in the network. The aggregation gain is directly proportional to the aggregation delay and size of the aggregation queue used for storing data packets.

Event notification time- ENT: It is the amount of time taken by a node to report data of a sensed event to final destination (sink). The minimum time taken by the nodes to report a sensed event represents best ENT and worst ENT refers to the maximum time taken by data packet of a particular event to reach the final destination. Both the best and worst ENT depends on the network congestion and aggregation delay introduced at each aggregator.

Energy Consumption: Each sensor node consumes some energy for processing and communicating data among the neighbors. The energy consumed by the different components of a sensor node includes MPU (in active and low power mode) and Radio (for data transmissions and receptions).

\subsection{Aggregation Gain}

The aggregation gain depends on the waiting period assigned at each aggregator before the received data is forwarded to the final destination. The degree of aggregation depends on the number of data packets that an aggregator can buffer and it directly affects the aggregation gain. Figure 3 shows that number of packets aggregated in TDA, NDDA and RDA schemes. It is clear from the figure 3 that NDDA scheme achieves highest aggregation by aggregating eight data packets. NDDA technique aggregates atleast one data packet for each event except event having id $E_{4}$. The TDA scheme aggregates seven data packets and the event having id $E_{0}$ aggregates maximum (three) data packets. Random delay based aggregation has equivalent performance in terms of aggregation, but event reporting is higher than Collect protocol.

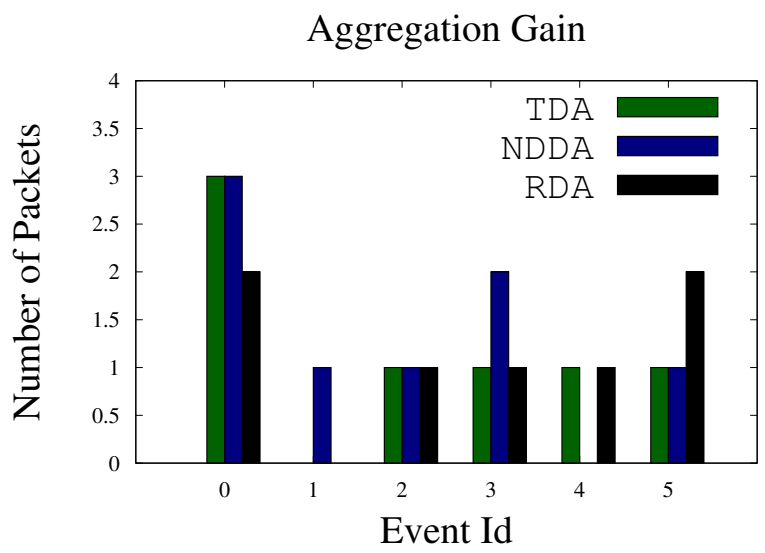

Fig. 3: The number of packets aggregated in NDDA, RDA and TDA techniques

\subsection{Event Notification Time}

The Tuned, Normalized and Random delay based aggregation schemes minimize the data traffic in the network and hence decreases the chances of congestion as compared to Collect protocol. 
The Tuned delay based scheme performs better than NDDA and RDA in terms of overall worst event notification time. The comparative analysis in terms of best and worst ENT of both the schemes is provided in the tables 1 and 2 . The tables indicate that the worst ENT of the TDA is better as compared to NDDA and Collect protocol. Tuned delay based aggregation scheme has comparable performance with Collect protocol in terms of ENT.

Table 1. : Comparative analysis of best ENT in TDA, NDDA, RDA and Collect protocol

\begin{tabular}{|c|c|c|c|c|}
\hline & \multicolumn{4}{|c|}{ Best ENT (seconds) } \\
\hline Event ID & TDA & NDDA & RDA & Collect \\
\hline$E_{0}$ & $\mathbf{0 5 . 0 5 6}$ & 08.056 & 03.057 & 04.055 \\
\hline$E_{1}$ & $\mathbf{2 8 . 5 6 9}$ & 28.430 & 15.929 & 06.430 \\
\hline$E_{2}$ & $\mathbf{2 1 . 8 0 7}$ & 43.806 & 48.306 & 37.307 \\
\hline$E_{3}$ & $\mathbf{4 3 . 9 3 1}$ & 46.556 & 19.805 & 40.680 \\
\hline$E_{4}$ & $\mathbf{5 2 . 1 8 1}$ & 49.431 & 56.557 & 48.306 \\
\hline$E_{5}$ & $\mathbf{0 5 . 1 8 1}$ & 11.931 & 06.430 & 08.432 \\
\hline
\end{tabular}

Table 2. : Comparative analysis of worst ENT in TDA, NDDA, RDA and Collect protocol

\begin{tabular}{|c|c|c|c|c|}
\hline & \multicolumn{4}{|c|}{ Worst ENT (seconds) } \\
\hline Event ID & TDA & NDDA & RDA & Collect \\
\hline$E_{0}$ & $\mathbf{4 3 . 3 0 5}$ & 45.679 & 28.307 & 57.431 \\
\hline$E_{1}$ & $\mathbf{5 2 . 5 5 6}$ & 62.806 & 55.681 & 47.431 \\
\hline$E_{2}$ & $\mathbf{3 3 . 5 5 6}$ & 48.307 & 74.306 & 58.682 \\
\hline$E_{3}$ & $\mathbf{5 2 . 8 0 5}$ & 46.556 & 36.430 & 59.569 \\
\hline$E_{4}$ & $\mathbf{5 9 . 6 8 2}$ & 66.057 & 58.556 & 59.805 \\
\hline$E_{5}$ & $\mathbf{2 8 . 0 5 6}$ & 42.306 & 30.444 & 49.180 \\
\hline
\end{tabular}

\subsection{Energy Consumption}

The main components of a sensor node that consume energy are MPU (mircocontroller) and radio. The MPU (active state) consume energy for processing data whereas the radio utilizes energy for communicating data over the network. The energy consumed by each node in different states such as MPU (both active and low power mode) and Radio (transmissions and receptions) is computed for NDDA, TDA, RDA schemes and Collect protocol. Figure 4 shows the energy consumed by MPU for TDA, NDDA, RDA schemes and Collect protocol. The comparative study elaborates that TDA scheme consumes significantly less energy than RDA and Collect protocol. From the figure 5 it has been observed that TDA scheme remains in low power mode for extended time and hence saves the overall energy. To communicate with other nodes, sensor nodes consume energy by transmitting and receiving data in the network. Figures 6 and 7 illustrate the energy consumed by the radio for data transmissions and receptions. It is abundantly clear from the figures 6 and 7 that the TDA performs better than NDDA, RDA and Collect protocol. Table 3 and figure 8 shows overall energy consumed in various states. From the figure 8 and table 3 , it has been observed that the overall performance of the TDA is much better than NDDA, RDA and Collect in terms of energy consumption.

\section{Energy Consumed by Radio-Transmissions}

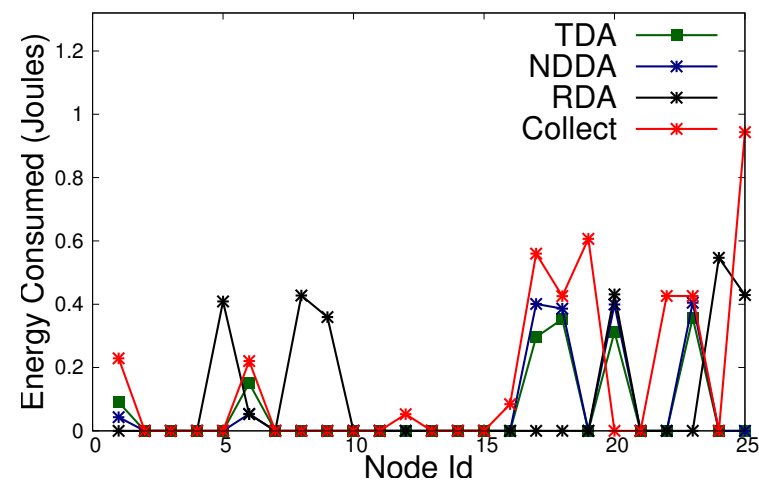

Fig. 6: Energy consumed by Radio for data transmissions in TDA, NDDA, RDA and Collect

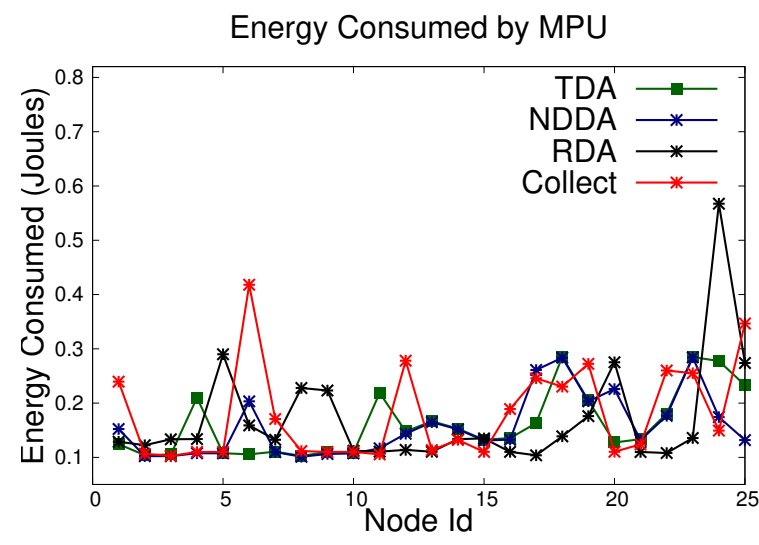

Fig. 4: Energy consumed by MPU in TDA, NDDA, RDA and Collect

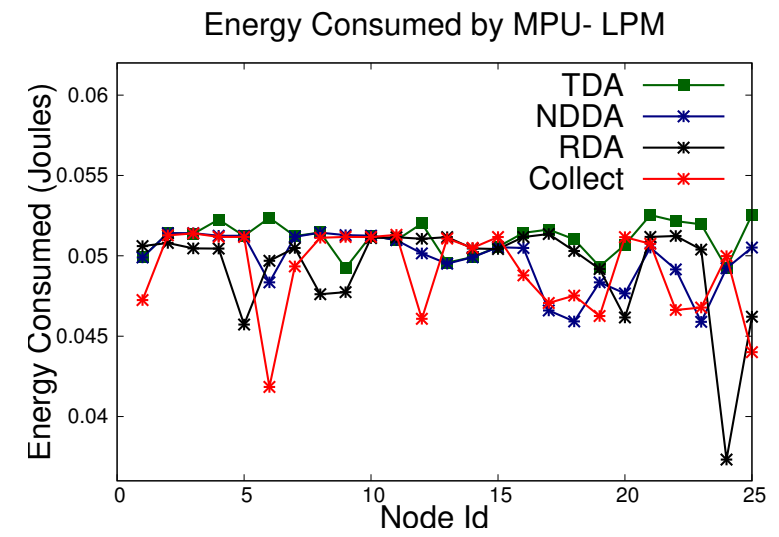

Fig. 5: Energy consumed by MPU in low power mode for TDA, NDDA, RDA and Collect 


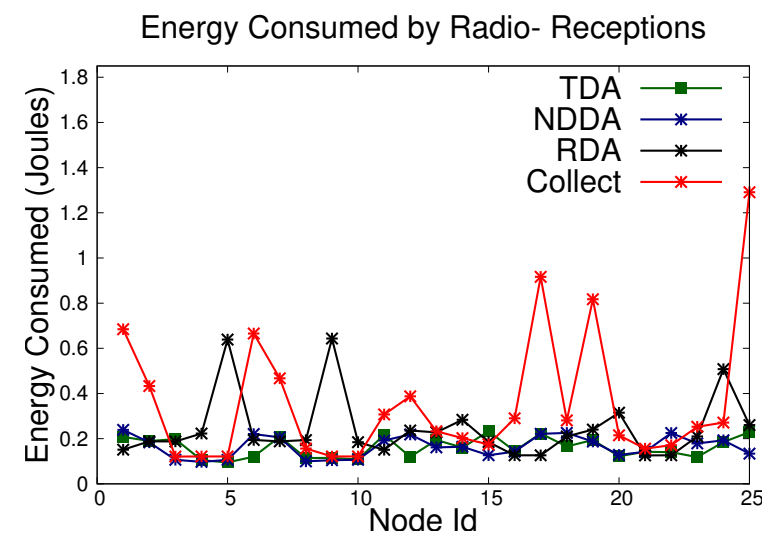

Fig. 7: Energy consumed by Radio for data receptions in TDA, NDDA, RDA and Collect

Total Energy Consumed

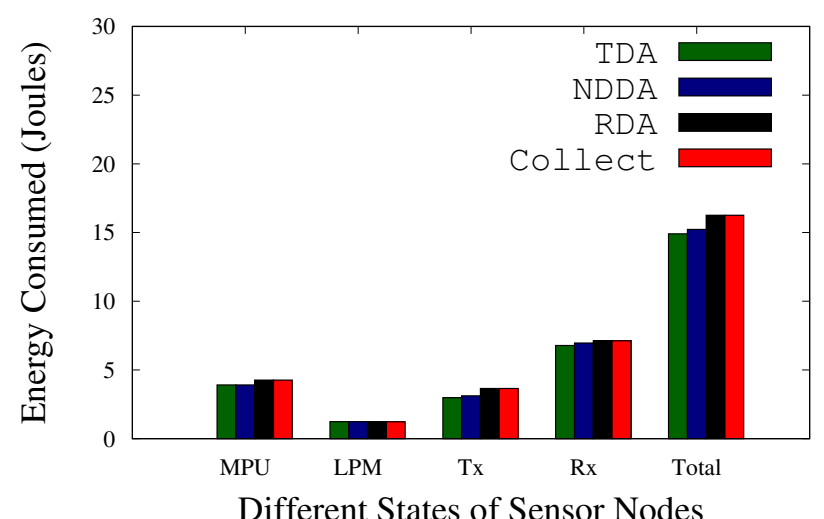

Fig. 8: Total energy consumption in TDA, NDDA, RDA and Collect in different states

Table 3. : Comparative analysis in terms of overall energy consumption in TDA, NDDA, RDA and Collect

\begin{tabular}{|l|c|c|c|c|}
\hline & \multicolumn{4}{|c|}{ Energy Consumption (Joules) } \\
\hline States & TDA & NDDA & RDA & Collect \\
\hline MPU (Active Mode) & $\mathbf{3 . 9 1}$ & 3.91 & 4.26 & 4.51 \\
\hline MPU (Low Power Mode) & $\mathbf{1 . 2 4}$ & 1.24 & 1.23 & 1.22 \\
\hline Radio (Transmissions- Tx) & $\mathbf{3 . 7 8}$ & 3.85 & 3.95 & 3.97 \\
\hline Radio (Receptions- Rx) & $\mathbf{7 . 3 8}$ & 7.52 & 7.72 & 8.97 \\
\hline Total & $\mathbf{1 6 . 3 1}$ & 16.52 & 17.16 & $\mathbf{1 8 . 6 7}$ \\
\hline
\end{tabular}

\section{CONCLUSION AND FUTURE DIRECTIONS}

In this paper, Tuned, Normalized and Randomized delay based structure free aggregation techniques are evaluated and investigated for performance by using various parameters like aggregation gain, energy consumption and event notification time. Through extensive simulations, it has been observed that tuned delay based aggregation scheme performs better than NDDA, RDA and Collect protocol in terms of worst ENT and energy consumption. From the results, it is clear that Tuned delay based scheme aggregates 32\% data and reduces energy consumption (upto 13\%) as compared to Collect protocol. TDA scheme also decreases worst event notification time (upto 20\%) in comparison to RDA aggregation scheme. This work can be extended by using some other network parameters for introducing the aggregation delay. Furthermore, there is a need to optimize the energy consumption and event notification time as there is trade-off between these two parameters.

\section{REFERENCES}

[1] I. F. Akyildiz, W. Su, Y. Sankarasubramaniam, and E. Cayirci. A survey on sensor networks. IEEE Communication Magazine, 40(8):102-114, 2002.

[2] M. Buettner, G. V. Yee, E. Anderson, and B. R. Han. X-mac: a short preamble mac protocol for duty-cycled wireless sensor networks. In 4th international conference on embedded networked sensor systems (SenSys-06), pages 307 - 320, 2006.

[3] C. M. Chao and T. Y. Hsiao. Design of structure free and energy balanced data aggregation in wireless sensor networks. Journal of Network and Computer Applications, 37:229-239, 2014.

[4] K. H. Chen, J. M. Huang, and C. C. Hsiao. Chiron: an energyefficient chain-based hierarchical routing protocol in wireless sensor networks. In Proceeding of IEEE Symposium on Wireless Telecommunications (WTS-2009), pages 1-5, Prague, 2009.

[5] S. Dietzel, B. Bako, E. Schoch, and F. Kargl. A fuzzy logic based approach for structure-free aggregation in vehicular adhoc networks. In Proceeding of 6th ACM International Workshop on Vehicle Networking (VANET-09), pages 79-88, USA, 2009.

[6] M. Ding, X. Cheng, and G. Xue. Aggregation tree construction in sensor networks. In Proceeding of 58th IEEE Vehicular Technology Conference, volume 4, pages 2168-2172, USA, 2003.

[7] A. Dunkels. The ContikiMAC Radio Duty Cycling Protocol. Technical report, Swedish Institute of Computer Science, 12 2011.

[8] A. Dunkels, F. Osterlind, N. Tsiftes, and Z. He. Softwarebased on-line energy estimation for sensor nodes. In Proceedings of the 4th workshop on Embedded networked sensors (EmNets-07), pages 28-32, USA, 2007.

[9] K. W. Fan, S. Liu, and P. Sinha. Structure-free data aggregation in sensor networks. IEEE Transactions on Mobile Computing, 6(8):929-942, 2007.

[10] O. Gnawali, R. Fonseca, K. Jamieson, M. Kazandjieva, D. Moss, and P. Levis. Ctp: An efficient, robust, and reliable collection tree protocol for wireless sensor networks. ACM Transactions on Sensor Networks, 10(1):1-50, 2013.

[11] O. Gnawali, R. Fonseca, K. Jamieson, D. Moss, and P. Levis. Collection tree protocol. In Proceedings of the 7th ACM Conference on Embedded Networked Sensor Systems (Sensys-09), pages 1-14, USA, 2009.

[12] J. Yuhui H. Junping and D. Liang. A time-based cluster-head selection algorithm for leach. In IEEE Symposium on Computers and Communications, pages 1172-1176, 2008. 
[13] W. B. Heinzelman, A. P. Chandrakasan, and H. Balakrishnan. An application-specific protocol architecture for wireless microsensor network. IEEE Transactions on Wireless Coтmunications, 1(4):660-670, 2002.

[14] R. Jurdak, P. Baldi, and C. V. Lopes. Adaptive low power listening for wireless sensor networks. IEEE Transactions on Mobile Computing, 6(8):988-1004, 2007.

[15] N. Kaur, S. S. Sran, and L. Kaur. Berp: Balanced energy routing protocol for routing around connectivity holes in wireless sensor networks. In IEEE International Conference on Recent Advances in Engineering and Computational Sciences (RAECS-2015), pages 1 - 6, India, 2015.

[16] P. Levis, N. Patel, D. Culler, and S. Shenker. Trickle: A selfregulating algorithm for code propagation and maintenance in wireless sensor networks. In Proceeding of International Conference on Networked Systems Design and Implementation (NSDI-04), volume 1, pages 2-2, USA, 2004.

[17] S. J. Lim and M. S. Park. Energy-efficient chain formation algorithm for data gathering in wireless sensor networks. International Journal of Distributed Sensor Networks, 2012:1-9, 2012.

[18] S. Lindsey and C. Raghavendra. Pegasis: Power efficient gathering in sensor information systems. In Proceeding 15th International Symposium on Parallel and Distributed Processing, pages 2001-2008, 2001.

[19] S. Lindsey, C. Raghavendra, and K. M. Sivalingam. Data gathering algorithms in sensor networks using energy metrics. IEEE Transactions on Parallel and Distributed Systems, 13(9):924-935, 2002.

[20] V. Loscri, G. Morabito, and S. Marano. A two levels hierarchy for low energy adaptive clustering hierarchy. In 62nd vehicular technology conference, pages 1809-1813, 2005.

[21] C. J. Merlin and W. B. Heinzelman. Schedule adaptation of low-power-listening protocols for wireless sensor networks. IEEE Transactions on Mobile Computing, 9(5):672685, 2009.

[22] Moteiv Corporation. Ultra low power IEEE 802.15.4 compliant wireless sensor module.

[23] E. F. Nakamura, H. A. B. F. de Oliveira, L. F. Pontello, and A. A. F. Loureiro. On demand role assignment for event-detection in sensor networks. In 11th IEEE Symposium on Computers and Communications (ISCC-06), pages 1-7, 2006.

[24] G. J. Pottie and W. J. Kaiser. Wireless integrated network sensors. Communications of the ACM, 43(5):51-58, 2000.

[25] J. Shin and C. Sun. Creec: Chain routing with even energy consumption. Journal of Communications and Networks, 13(1):17-25, 2011.

[26] S. S. Sran, L. Kaur, G. Kaur, and S. K. Sidhu. Energy aware chain based data aggregation scheme for wireless sensor network. In IEEE International Conference on Energy Systems and Applications (ICESA-2015), pages 113 - 117, India, 2015.

[27] S. S. Sran, J. Singh, and L. Kaur. Aggregation aware early event notification technique for delay sensitive applications in wireless sensor networks. International Journal of Sensor Networks, Aceepted for publication, 2017.

[28] N. Tabassum, Q. Ehsanul, K. Mamun, and Y. Urano. Cosen: A chain oriented sensor network for efficient data collection.
In Proceeding of 3rd IEEE international Conference on Information Technology, New Generations: ITNG 2006, volume 4, pages 262-267, USA, 2006.

[29] H. O. Tan and I. Korpeoglu. Power efficient data gathering and aggregation in wireless sensor networks. ACM Sigmod Record, 32(4):66-71, 2003.

[30] L. A. Villas, A. Boukerche, H. A. B. F. de Oliveira, R. B. de Araujo, and A. A.F. Loureiro. A spatial correlation aware algorithm to perform efficient data collection in wireless sensor networks. Ad Hoc Networks, 12:69 - 85, 2014.

[31] L. A. Villas, Azzedine Boukerchel, Regina Borges de Araujo, and Antonio A. F. Loureiro. Highly dynamic routing protocol for data aggregation in sensor networks. In Proceedings of the IEEE Symposium on Computers and Communications, pages $496-502,2010$.

[32] L. A. Villas, D. L. Guidoni, R. B. Araujo, A. Boukerche, and A. A. F. Loureiro. A scalable and dynamic data aggregation aware routing protocol for wireless sensor networks. In Proceedings of the 13th ACM international conference on Modeling, analysis and simulation of wireless and mobile systems (MSWIM-10), pages 110 - 117, USA, 2010.

[33] H. Yousefi, M. H. Yeganeh, N. Alinaghipour, and A. Movaghar. Structure-free real-time data aggregation in wireless sensor networks. Computer Communications, 35(9):1132-1140, 2012.

[34] J. Zhang, Q. Wu, F. Ren, T. He, and C. Lin. Effective data aggregation supported by dynamic routing in wireless sensor networks. In Proceeding of IEEE Communications (ICC2010), pages $1-6$, Cape Town, 2010. 